\title{
ECG Web Services for Patient Health Monitoring in Internet of Things
}

\author{
Jyoti L. Khachane \\ Student of M.E. Computer \\ Computer Department, DYPIET, Pimpri.
}

\author{
Latika R. Desai \\ Assistant Professor \\ Computer Department DYPIET, Pimpri
}

\begin{abstract}
Now a day people are facing many health related problems. There is large amount of data that is not structured. The gathered information is raw and unstructured in the nature, so there is a need of converting it to meaningful services. In this paper such system is developed which will provide web service that will be available to any other person easily. Every person should get the healthcare services anywhere, every time even at their home also. For this purpose a platform is developed so that patient specially senior citizens can know their electro Cardiogram (ECG) or also can be said as heart rate, oxygen rate, temperature rate, through an android mobile application specially developed for the healthcare. This overall concept is developed under the Internet of Things as well as cloud computing environment. App which is developed named as 'Patient Health Monitoring'. This paper gives an idea about Adriano kit which used in the project. This paper also gives an idea about the sensors which are used to measure above mentioned measures. A Bluetooth sensors is also used here for the connectivity. Server will be any network connection such as Wi-Fi.
\end{abstract}

\section{Keywords}

Web Service Discovery, Restful Web Services, Internet of Things, ECG, Arduino kit.

\section{INTRODUCTION}

The Internet of Things (IoT) is the network of physical objects or things embedded with electronics, software, sensors, and network connectivity, which enables these objects to collect and exchange data. The Internet of Things allows objects to be sensed and controlled remotely across existing network infrastructure creating opportunities for more direct integration between the physical world and computer-based systems, and resulting in improved efficiency, accuracy and economic benefit. Each thing is uniquely identifiable through its embedded computing system but is able to interoperate within the existing Internet infrastructure. Experts estimate that the IoT will consist of almost 50 billion objects by 2020. IoT is expected to offer advanced connectivity of devices, systems, and services that goes beyond machine-tomachine communications (M2M) and covers a variety of protocols, domains, and applications. The interconnection of these embedded devices (including smart objects), is expected to usher in automation in nearly all fields, while also enabling advanced applications like a Smart Grid and expanding to the areas such as smart cities. Web services are independently developed applications that are exposed as services and interconnected using Web network infrastructure with standards such as XML, UDDI, SOAP and WSDL. In the proposed system patients can get interaction with the doctor and also make doctor to check their health information which obtained through the sensors used. The data collected by sensors is initially in unstructured format, so here DBMS is used for storing that data in well-structured format using MYSQL for accessing that data.

\section{MOTIVATION}

The Internet of Things is a new era of intelligence computing and its providing a privilege to communicate around the world. The objective of IoT is Anything, Anyone, Anytime, Anyplace, Any service and any network. Every day the modern people expect new device and new technology to simplify their day to day life. It can become possible by discovering \& grouping the desired web services by forming a cluster here RESTfull web services are used to design the web services. In todays' environment people are facing a lot of problems with their health. Sometime it is inconvenient to the patient especially senior citizens to go to the hospital due to accommodation or any health related problems.

\section{OBJECTIVES}

The major objectives for this projects are

1. To develop a mechanism for service creation as well as for service discovery.

2. To implement a lightweight approach for cluster discovery.

3. To develop an access control mechanism for IoT environment.

4. To observe the patient continuously from remote place also.

To discover and cluster lightweight, simple, semantic web services an approach is introduced based on Electrocardiogram web services that will serves better for people,those are facing problem regarding on health issues in their area. Those are economically in poor condition.

\section{ARCHITECTURE}

The figure 1 shows the proposed system architecture which works on two side such as on doctor side \& patients' side. The hardware requirement $\&$ software requirement are as follows. 

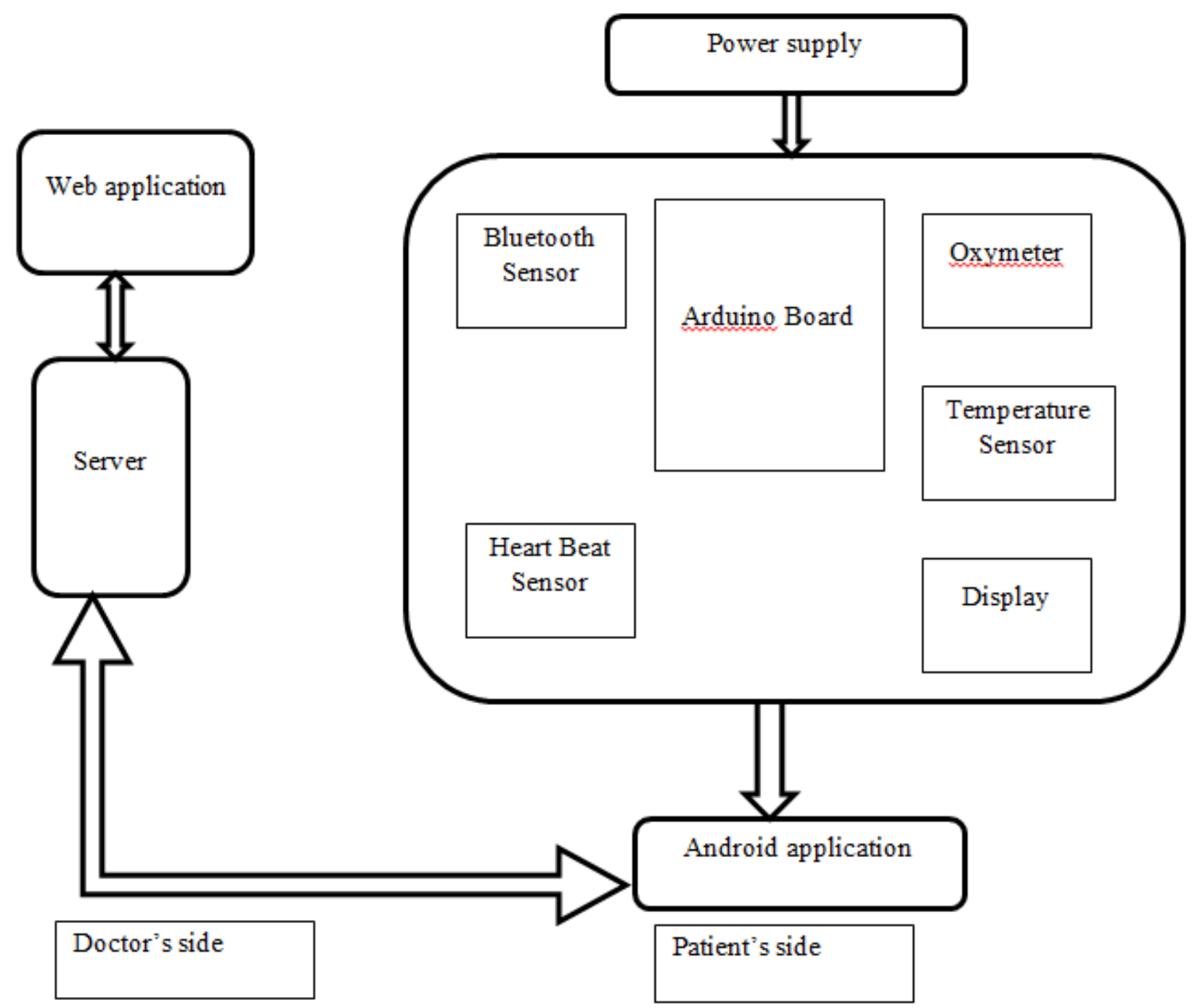

Fig 1: Proposed system architecture.

1) Hardware requirement :

i) Medical Sensor: To measure the patient's parameters

ii) Temperature sensor

iii) Oxymeter

iv) Heart beat sensor

v) Arduino board is used here with Bluetooth sensor.

vi) Android mobile with application.

\section{2) Software requirement}

i) A permanent web connection will be required.

ii) Any web browser with specified web application.

\section{WORKING OF SYSTEM}

The system is based on portable and mobile devices and wireless sensor networks for real time monitoring and analysis of various parameters of patients. This system is aimed at developing which can facilitate the diagnosis for the doctors through remote monitoring of patients. A set of medical and environmental sensors are used to monitor the health as well as the environment of the patient. This sensor data is then sending to the server using a mobile device. The doctors and caretakers monitor the patient in real time through the data received through the server. System is developed in such way that when web application will be run on the PC then android application will also work i.e. login or register is possible only when user will run web application on the server. Here Eclipse indigo tool is used which support java platform. The overall information will be stored in database. User can access that information when needed in future. Bluetooth sensor used HC06 will send the values that are collected by sensors to the android application when hotspot of the mobile will be on which will connect whole components like a single network. The medical history of each patient is stored on cloud storage for easy access and processing for logistics and prognosis of future complications. The architecture is so designed for monitoring patient privately at home as well as multiple patients in hospitals and public health care units. The result will be shown in application installed on android phone. It will show temperature of body, heart beat rate, and oxygen level of the body.

\subsection{Input to the System:}

Raw data gathered from different sensors and calculated frequency rates should be used as parameters

E.g. Body Temperature, Blood Pressure, Heartbeat etc. 


\subsection{Output from the System}

Frequency rates should be displayed over mobile and by analyzing these frequency rates prescription should be given by doctor.

E.g. Body and Environment Temperature level, Blood Pressure Level, Heartbeat count etc.

Prescription E.g. if Temperature is higher than normal the patient is suffering from fever so doctor should suggest prescription and medication for Fever.

\subsection{Generalized Working Of Sensors}

\subsubsection{Heart beat sensor:}

Heart beat sensor is designed to give digital output of heat beat when a finger is placed on it. When the heart beat detector is working, the beat LED flashes in unison with each heart-beat. This digital output can be connected to microcontroller directly to measure the Beats Per Minute (BPM) rate. It works on the principle of light modulation by blood flow through finger at each pulse.

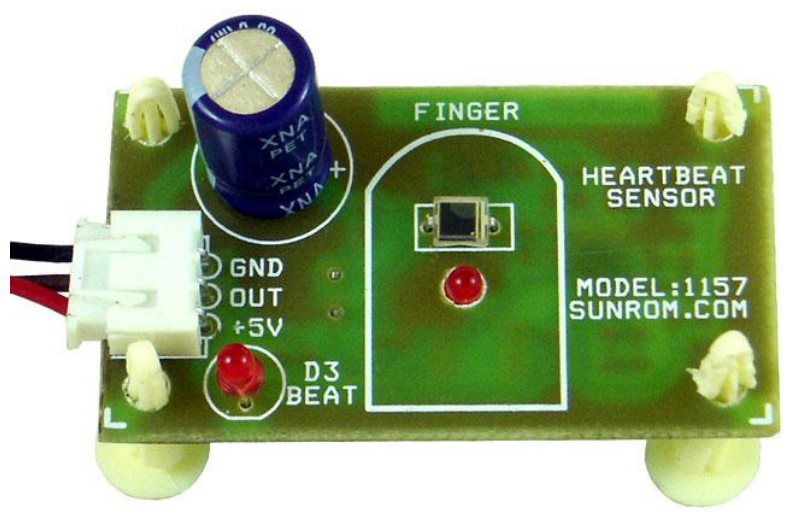

Fig2. Heart beat sensor

\subsubsection{Temperature sensor}

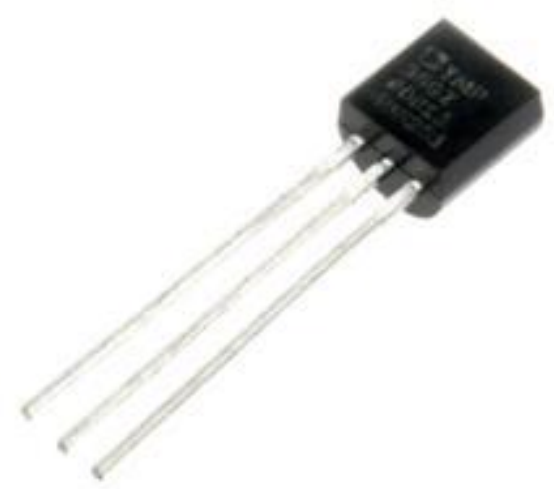

Fig3. Temperature sensor LM35

Temperature sensor shown in figure 3 has three terminals and required maximum of $5.5 \mathrm{~V}$ supply. This change of resistance is sensed by circuit and it calculates temperature. Temperature increases as voltage increases. This operation can be seen by using diode. There is one type of material that performs operations according to the temperature that varies to the resistance.

\subsubsection{Blood pressure sensor}

Oxymeter is used to measure blood pressure. This device clips patient's finger and measures heart rate and percentage of oxygen in patient's blood. It is designed for detecting hypoxia which is the condition caused by insufficient oxygen. The hemoglobin in our blood consists of oxygen that is circulated through our blood. Oxymeter emits infrared light that shine through our body's tissues to photo sensor resides on other side. Infrared light detects amount of hemoglobin that is saturated with oxygen. Oxymeter reading above $90 \mathrm{~s}$ is considered normal.

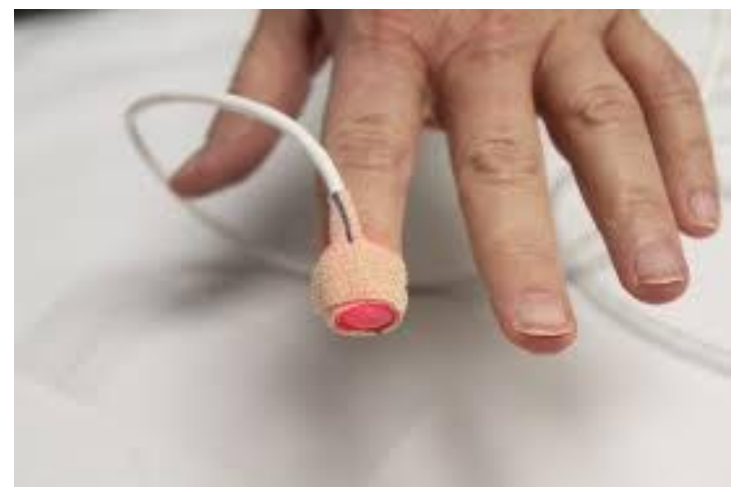

Fig4 Blood pressure sensor (oxymeter)

\section{CONCLUSION AND FUTURE SCOPE}

Many people from remote places have no any other way to daily for frequently communicate with doctor. They can't visit to hospital. So this android mobile app may help such people to give report of their physical conditions and this report or data will be read by doctor and prescription as well as health related suggestions will be given to patients. With using application and having permanent net connection each user can have benefit of the system. All sensors are reliable in case of security \& accuracy perspectives. So the reliability of the system gets increases. In today's life user can't pay attention to their health so by making this system more advance for user so that each person can be benefitted from this system. In future by adding more services that are essential for the user the future perspectives can be achieved here.

\section{RESULT}

The result will be shown in android application as well as on the display. Figure 4 shows the result generated by temperature sensor LM35. If any person has hold the sensor in two fingers then only it will show body temperature otherwise it will show room temperature present at that time.

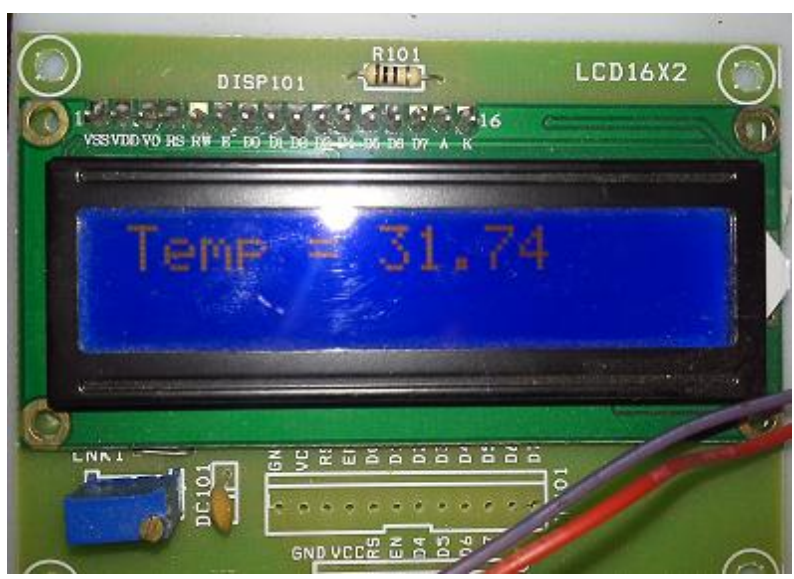

Fig4. Result of temperature sensor on display 
Figure 5 shows the result generated by heart beat sensor aswell as blood pressure sensor i.e. oxymeter which shows the oxygen level present in the blood at that time. In case of heart rate, the pulse counts measured by sensor which consist of led light which passes through skin and diode that sense the reflected light, according to that it shows the heart beat count for that time.

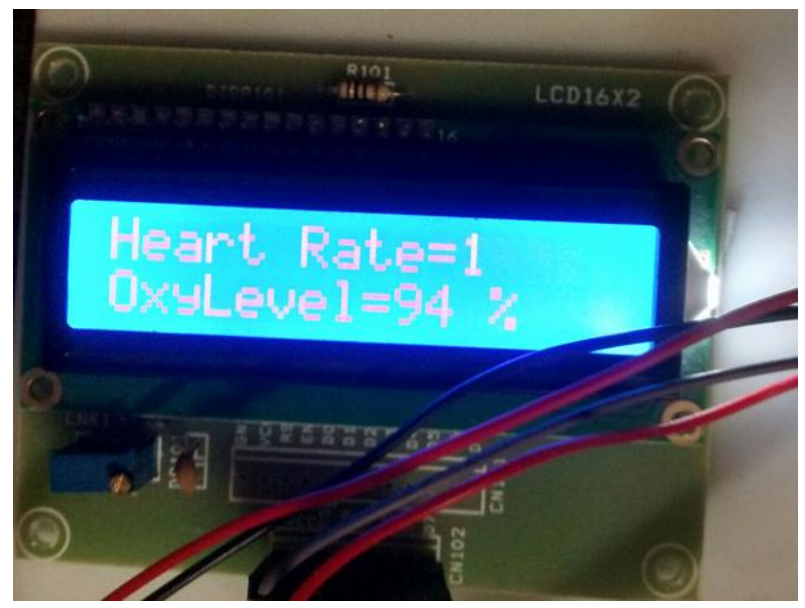

Fig5. Result of heart rate sensor \& blood pressure sensor on display

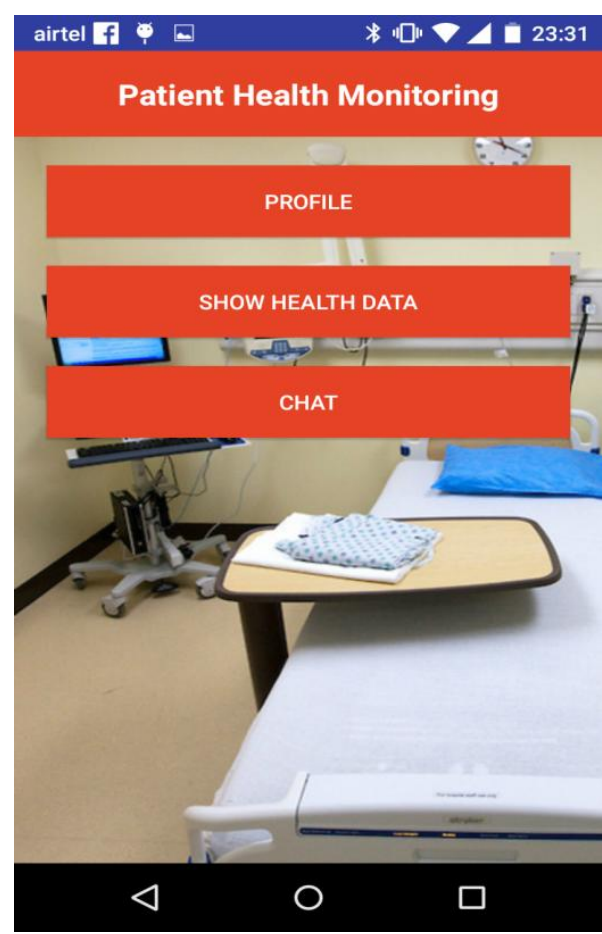

Fig6. Screenshot of Android Application

The app developed is Patient Health Monitoring App. When user $\log$ in to the system it shows profile of the user if user want to update it. Show health data shows reading of the patient's body that is collected from the sensors on the kit. It is shown in the fig7. This indicates oxygen level, body temperature and heart rate of the user. By using chat option user can chat with doctor on health related issues.

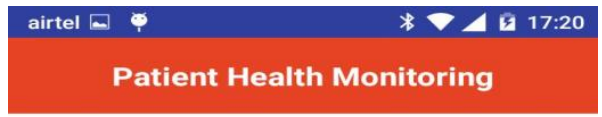

Oxygen level: $95 \%$

Body temperature: 26.37

Heart Rate: 1

\section{$\triangleleft$}

○

$\square$

Fig7. Screenshots of Android App Showing Health Data
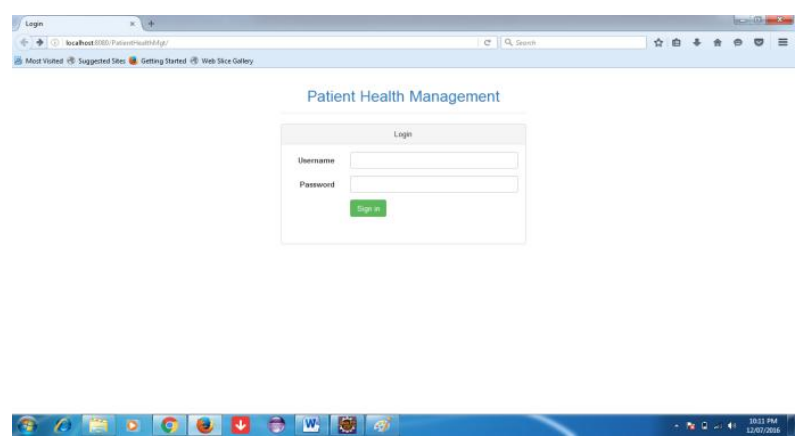

Screenshot 1 Admin's login form

$+$

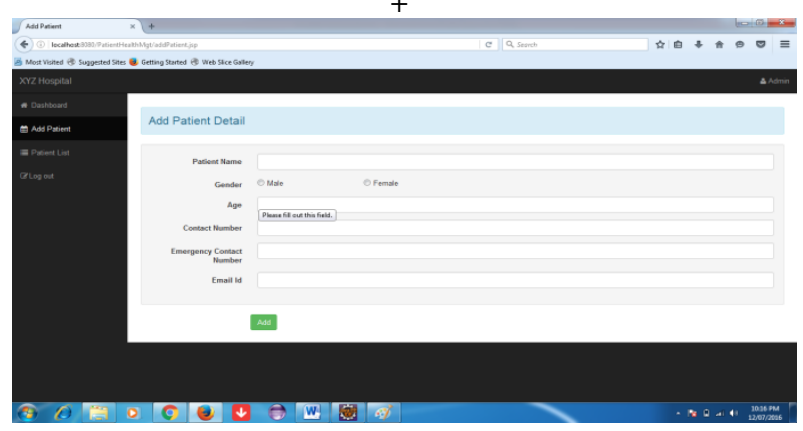

Screenshots 3. Registration of new patients

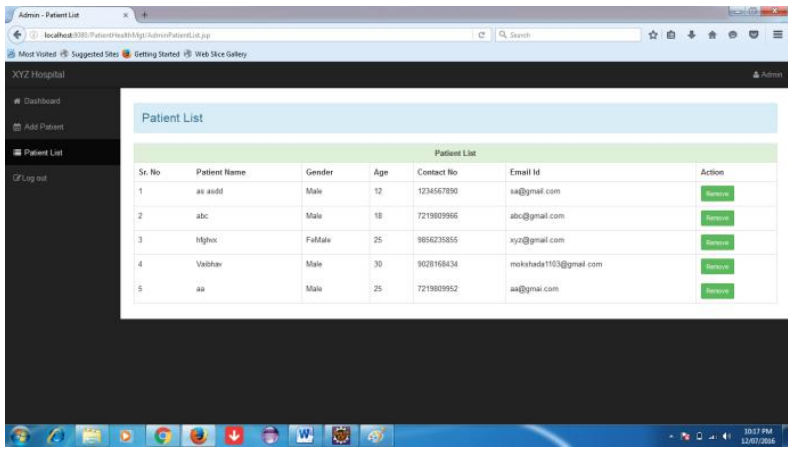

Screenshots 4. Patients' details 


\section{REFERENCES}

[1] Zhang, Lili, et al. "Research on IOT RESTful Web Service Asynchronous Com-position Based on BPEL." Intelligent Human-Machine Systems and Cybernetics(IHMSC), 2014 Sixth International Conference on. Vol. 1. IEEE, 2014.

[2] Gao, Ruiling, et al. "Web-based motion detection system for health care."Computer and Information Science (ICIS), 2015 IEEE/ACIS 14th International Conference on. IEEE, 2015.

[3] Wu, Hang, and Chaozhen Guo. "The research and implementation of Web Service classication and discovery based on semantic." Computer Supported Cooperative Work in Design (CSCWD), 2011 15th International Conference on IEEE, 2011.

[4] Artail, Hassan, Kassem Fawaz, and Ali Ghandour. "A proxy-based architec- ture for dynamic discovery and invocation of web services from mobile devices."Services Computing, IEEE Transactions on 5.1 (2012): 99-115.

[5] Rong, Wenge, and Kecheng Liu. "A survey of context aware web service discovery: from user's perspective."
Service Oriented System Engineering (SOSE) 2010 Fifth IEEE International Symposium on. IEEE, 2010.

[6] A.R. Hevner, D. Zhang, and J.L. Zhao, Guest Editorial: Introduction to the Special Issue on Modeling and Implementation of Service Enterprise Systems, IEEE Trans. Serv. Comput., vol. 3, no. 2, pp. 86-88, Apr./June 2010.

[7] Q. Yu, X. Liu, A. Bouguettaya, and B. Medjahed, Deploying and Managing Web Services: Issues, Solutions, and Directions, VLDB J., vol. 17, no. 3, pp. 537-572, May 2008

[8] M. Huhns and M. Singh, Service-Oriented Computing: Key Concepts and Principles, IEEE Internet Comput., vol. 9, no. 1, pp. 75-81, Jan./Feb. 2005.

[9] E. Fleisch and F. Mattern, Das Internet der Dinge: Ubiquitous Computing and RFID in derPraxis: Visionen,Technologien,Anwendungen,Handlungsanleitu ngen. Springer-Verlag, 2005.

[10] Deng Ziyun, SOA Practitioners: System Integration in Distributed Environment. 